\title{
Development and Validation of Multi-Residue Analysis of 82 Pesticides in Grapes and Pomegranate as per the Requirements of the European Union (EU) and Codex Alimentarius Using GC-MS/MS with Compound Based Screening
}

\author{
Gouri Satpathy $^{1}$, Yogesh Kumar Tyagi ${ }^{1, *}$, Rajinder Kumar Gupta ${ }^{2}$ \\ ${ }^{1}$ University School of Basic and Applied Sciences, GGS Indraprastha University, Delhi, India \\ ${ }^{2}$ University School of Biotechnology, GGS Indraprastha University, Delhi, India \\ *Corresponding author: drytyagi@gmail.com
}

Received September 30, 2013; Revised February 25, 2014; Accepted March 12, 2014

\begin{abstract}
The Quick Easy Cheap Effective Rugged and Safe (QuEChERS) multiresidue method has been validated for the extraction of 82 pesticides belonging to various chemical classes from grapes and pomegranate (commodities with high sugar and low lipid contents). A mixture of 82 pesticides amenable to gas chromatography (GC) was quantitatively recovered from spiked grapes and pomegranate and determined using gas chromatographytandem mass spectrometry (GC-MS/MS). The method employed involved initial extraction in a water/ethyl acetate system, an extraction/partitioning step after the addition of salt, and a cleanup step utilizing dispersive solid-phase extraction (d-SPE); this combination ensured that it was a rapid, simple and cost-effective procedure. The method setup is streamlined with the new software approach of Compound Based Scanning (CBS). The matrix-matched calibration results have demonstrated good reproducibility, robustness and linearity. The spiking levels for the recovery experiments were $0.005,0.01$ and $0.1 \mathrm{mg} \mathrm{kg}^{-1}$ for GC-MS/MS analyses. Adequate pesticide quantification and identity confirmation were attained, even at the lowest concentration levels, considering the high signal-to-noise ratios, the very good accuracies and precisions, as well as the good matches between the observed ion ratios. Mean recoveries mostly ranged between 70 and $110 \%$ (91\% on average), and RSD were generally below $12 \%$ (7.3\% on average). The use of analyte protectants during GC analysis was demonstrated to provide a good alternative to the use of matrix-matched standards to minimize matrix-effect-related errors. For all compounds LODs were 0.001 to $0.005 \mathrm{mgkg}^{-1}$ and LOQs were 0.005 to $0.020 \mathrm{mgkg}^{-1}$. Correlation coefficients of the calibration curves were $>0.991$. Based on these results, the methodology has been proven to be highly efficient and robust and thus suitable for monitoring the Maximum Residual Limit (MRL) compliance of a wide range of commodity/pesticide combinations.
\end{abstract}

Keywords: grapes, pomegranate, pesticides, QuEChERS, method validation, GC-MS/MS

Cite This Article: Gouri Satpathy, Yogesh Kumar Tyagi, and Rajinder Kumar Gupta, "Development and Validation of Multi-Residue Analysis of 82 Pesticides in Grapes and Pomegranate as per the Requirements of the European Union (EU) and Codex Alimentarius Using GC-MS/MS with Compound Based Screening." American Journal of Food Science and Technology, vol. 2, no. 2 (2014): 53-61. doi: 10.12691/ajfst-2-2-2.

\section{Introduction}

The term "pesticides" is commonly used to describe a very broad class of crop-protecting chemicals, including insecticides, herbicides and fungicides. Together, these compounds have become a primary tool in modern agriculture and have contributed to a dramatic increase in crop yields in recent decades for most field, fruit, and vegetable crops. Along with the obvious benefits, concerns have also surfaced regarding the effect of these chemicals on human health. Considering the lethal effects of pesticides on human health, their residues in food commodities are issues of public concern and are regulated by several legislations. In order to address these concerns, governments have initiated monitoring programs to enforce regulatory compliance and ensure food safety. Given that pesticides are polluting the earth and causing problems in human beings and wildlife, the quantity of pesticide being consumed becomes a necessary knowledge. Pesticide residue analysis is an important process in determining the safety inusing certain pesticides.

Grapes and pomegranates are important fruit crops in India, the commercial cultivation of which receives frequent application of a large volume of pesticides throughout the cropping season to control a variety of 
pests and diseases. Pesticide residue is a major concern for the stakeholders of the grape industry, since quality regulations and food safety standards are becoming more stringent in most countries. The management of pesticide residues in grapes is challenging because, besides direct application, pesticide residues may also appear in grape berries from indirect sources like soil, contaminated agroinputs (e.g. manures, fertilizers, growth regulators, irrigation water, etc.), drift from adjoining fields of other agricultural crops, etc. Hencehundreds of chemicals are regularly monitored in the grape and pomegranate samples of Indian origin for export, although the number of recommended chemicals for direct use in grape cultivation is only 45 [1]. Considering the importance of food safety, the Government of India has made a satisfactory certificate of residue analysis a mandatory prerequisite for issuance of a phytosanitary certificate for export [2]. Therefore analysis of pesticide residues is an important process to determine the safety of using certain pesticides. Consequently, governments, food producers and food retailers have a duty to ensure that any residues occurring in the foods for human consumption are at or below statutory MRLs. Europe and US, among others, look for certified quality produce for import and have stringent agricultural practices and are particular about residue content. Commission decision 90/642/EEC adopted in the European Union sets MRLs for more than 500 different pesticides in over 300 different food commodities. Many of these MRLs are set at a default value of $0.01 \mathrm{mgkg}^{-1}$, the typical limit of determination of routine analytical methods. Thus there is requirement to have an analytical test method to analyze grapes and pomegranates for large numbers of pesticides at concentrations at or below 0.01 $\mathrm{mgkg}^{-1}$, preferably with low costs and rapid analysis (rapid turnaround times $<24 \mathrm{~h}$ ). This is not possible with single Quadrupole MS and is often achieved using MRM based on the use of a combination of LC-MS/MS and GCMS/MS due to the most selective and sensitive nature of the detectors $[3,4,5,6,7,8]$.

Every good detection technique requires purified sample that can meet quality requirements for any particular analysis. It also requires a good sample preparation technique (high sample throughput, ruggedness, ease-of-use, low cost and labor, minimal solvent usage and waste generation, occupational and environmental friendliness, small space requirements and minimal material and glassware needs) and needs to be suitable for both single processing as well as batch processing. A number of methods have been reported for extraction of pesticide residues in fruits and vegetables, which involve an aqueous acetonitrile extraction with dSPE cleanup and GC-MS/MS detection. However, they were not in total compliance with the criteria established by the EU [9]. Although acetonitrile based extraction is readily compatible with LC-MS/MS, certain issues are encountered when less polar semi-volatile pesticides are analyzed by GC-MS/MS. Direct analysis of acetonitrile by GC is not compatible and can degrade the GC column phase. It also leads to poor focusing of chromatographic peaks because the vapour created by the sample overloads the insert liner dimension due to a high thermal expansion coefficient [10,11]. Moreover, evaporation and reconstitution of acetonitrile can cause loss of concentration of some of organophosphorous analytes such as monocrotophos, phorate, etc. Another problem is the high cost and availability of acetonitrile in the market.

Hence, the main objective of the study was to develop an improved method for reliable identification and accurate quantification of pesticide residues in grapes and pomegranates. The methodology was based on prior work with enhancement of a few analytical properties. Some of the important aspects considered during the present work were: (i) extension of the scope of previous methods so as to include as many target analytes as possible, (ii) design and optimization of MRM for the GC-MS/MS acquisition method, and (iii) testing the effectiveness of alternate cheaper solvent (ethyl acetate) extraction followed by PSA and GCB clean-up as means of reducing adverse influence of co-extractants on method and instrument performance. In addition, an important part of this work was demonstrating the performance of the developed method as per section 1.8 and for regular analysis.

\section{Experimentals}

\subsection{Materials}

The composite samples, consisting of 1-2 kg of grapes and pomegranate were obtained from a local market, Delhi. The standard stock solutions (1000 ppm) of all the 82 pesticides listed in Table 1 were prepared in ethyl acetate and stored at $4^{\circ} \mathrm{C}$. Ethyl acetate was procured from RFCL, Delhi, India. Other chemicals: anhydrous magnesium sulfate, Primary Secondary Amine (PSA) and Graphatized Carbon Black (GCB)were from Agilent Technologies.

\subsection{Sample Preparation}

A modified QuEChERS method using ethyl acetate was used for the preparation of samples $[9,11,12]$. According to this method, $10 \mathrm{~g}$ of finely ground sub-sample was placed in a polypropylene centrifuge tube $(50 \mathrm{~mL}) .10 \mathrm{~mL}$ ethyl acetate was added to it and the mixture was shaken vigorously on a laboratory shaker for $2 \mathrm{~min}$ and then centrifuged at $3000 \mathrm{rpm}$ for $2 \mathrm{~min}$. For clean-up, an aliquot $(2 \mathrm{~mL})$ of the supernatant was transferred into a polypropylene centrifuge tube containing $100 \mathrm{mg}$ anhydrous magnesium sulphate, $40 \mathrm{mg}$ PSA and $10 \mathrm{mg}$ GCB. The tube was then vortexed for $1 \mathrm{~min}$ and centrifuged at $3000 \mathrm{rpm}$ for $2 \mathrm{~min}$. An aliquot of the supernatant was transferred into a glass vial for GCMS/MS analysis. The sample concentration in the final extracts was $1 \mathrm{gmL}^{-1}$.

\subsection{Instrumental Conditions}

GC analyses were performed using a Scion TQ-MS/MS gas chromatograph, equipped with electronic flow control (EFC), a 1079 universal capillary injector and a CP-8400 auto sampler. The injector temperature was maintained at $260^{\circ} \mathrm{C}$. $2 \mu \mathrm{l}$ injections were performed by split-splitless injections with the split ratio as follows: initially set at 20:1; then, the split vent was closed from $0.01 \mathrm{~min}$ to 1.5 min, after which the split ratio was held at 100:1 till the 20th min, and finally reduced to 20:1. A BR-5 ms (30m $\times$ $0.25 \mathrm{~mm}, 0.25 \mu \mathrm{m})$ column was used for separation. Helium (of $99.999 \%$ purity) was used as carrier gas at a 
flow rate of $1.5 \mathrm{mLmin}^{-1}$. The column was held at $90^{\circ} \mathrm{C}$ for $3 \mathrm{~min}$ after injection, then programmed at $20^{\circ} \mathrm{C}$ to $150^{\circ} \mathrm{C} \mathrm{min}{ }^{-1}$ and then to $300^{\circ} \mathrm{C}$ at $1^{\circ} \mathrm{C} \mathrm{min}^{-1}$ and was held for $4 \mathrm{~min}$ (total runtime $37 \mathrm{~min}$ ). In this study, GCMS/MS with an Axial Ion Source, Active-focusing q0, curved collision cell (for multiple noise cancellation), Extended Dynamic Range (EDR) detector (for analyzing samples at ppb to ppm level) and a new compound-based screening (CBS) approach was used. MS was operated in the EI mode at $-70 \mathrm{eV}$. A collect delay time of $5.95 \mathrm{~min}$ was set to prevent instrument damage. The temperatures of the transfer line, ion source, manifold, active focuses were set at $300,230,40$ and $135^{\circ} \mathrm{C}$, respectively. The MS was calibrated with perfluorotributylamine (PFTBA). For the MS/MS experiments, argon (99.9998\% purity) was used as the collision gas with the collision cell pressure set at 1.7 mTorr. Data acquisition and processing were performed using MS Workstation, version 8.0.

\subsection{Method Performance}

Method validation and uncertainty of measurement were estimated using international guidelines $[15,16]$. The linearity of the pesticide mixtures were prepared containing 82 compounds and the calibration curves were studied at a concentration range between 0.005 and 0.1 $\mathrm{mgkg}^{-1}$ with duplicate injections prepared in both blank matrix extract and in ethyl acetate. The accuracy and precision of the method was assessed using spiked samples of grapes and pomegranate for six replicates at three spiking levels of $0.01,0.05$, and $0.1 \mathrm{mgkg}^{-1}$ to evaluate efficiency of the method. The results were always calculated using matrix-matched multi-level calibration standards bracketing the samples. LODs of all tested analytes were taken where $\mathrm{S} / \mathrm{N} \geq 3$ and LOQs were taken as the lowest spiking level, at which the validation was achieved (i.e. the average recovery was in the range 70 $120 \%$ with RSD $\leq 20 \%$ ), and was determined based on the accuracy and precision data obtained through the recovery studies.

\section{Results and Discussion}

\subsection{Optimization of Sample Preparation and GC-MS/MS Acquisition Method}

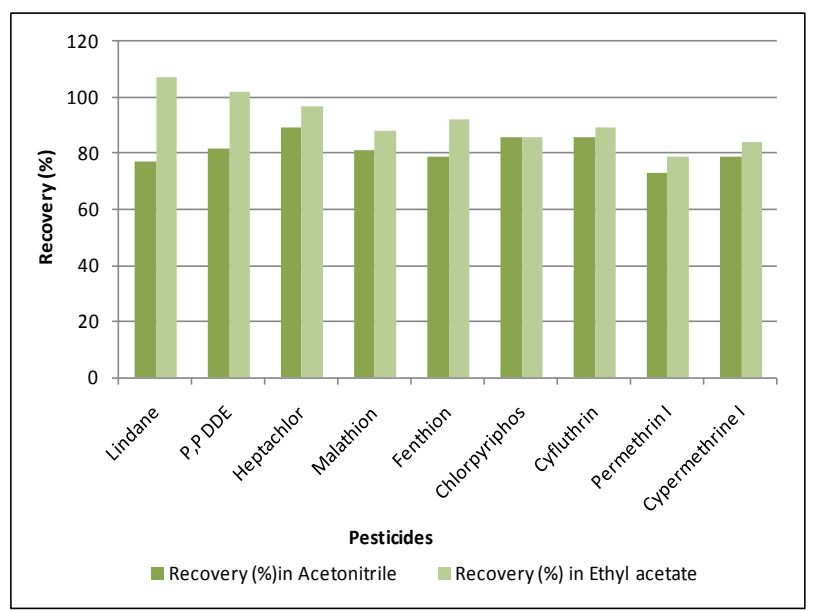

Figure 1. Comparison of recovery (\%) obtained from different solvent extract of grapes
In this method, a single and quick step of extraction using ethyl acetate was used because it is equally acceptable for extraction of non-fatty food, and especially suitable for the extraction of high-sugar commodities like grapes, since sugar has limited solubility in ethyl acetate $[13,14]$. Furthermore, substitution of acetonitrile by ethyl acetate increased the recovery of OC, OP and SP and it substantially reduces the input cost of analysis (Figure 1).

The purification step was optimized by using different weight of dSPE materials (PSA and GCB) to remove fatty acids and pigments from extracts of grapes and pomegranate. As a comparison of the results obtained from different weights of dSPE material in grapes extract, $40 \mathrm{mg}$ PSA and GCB of $10 \mathrm{mg}$ were found sufficient and gave equivalent response as of $100 \mathrm{mg}$ each (Figure 2). Therefore the above weights of PSA and GCB were selected for this experiment to remove interference from the matrix successfully and to reduce the cost of analysis.

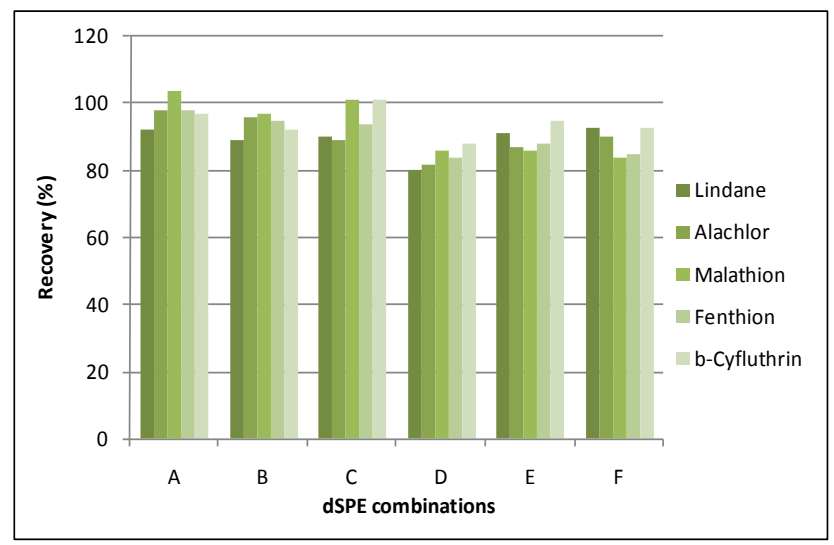

Figure 2. Comparison of recovery (\%) obtained from different weight of dSE of grapes ((A)100 mg of each PSA and GCB(B) $50 \mathrm{mg}$ of each PSA and GCB,(C) $40 \mathrm{mg}$ of each PSA and GCB (D) $30 \mathrm{mg}$ of each PSA and GCB (E) $40 \mathrm{mg}$ PSA and $20 \mathrm{mg}$ GCB (F) $40 \mathrm{mg}$ PSA and $10 \mathrm{mg}$ GCB
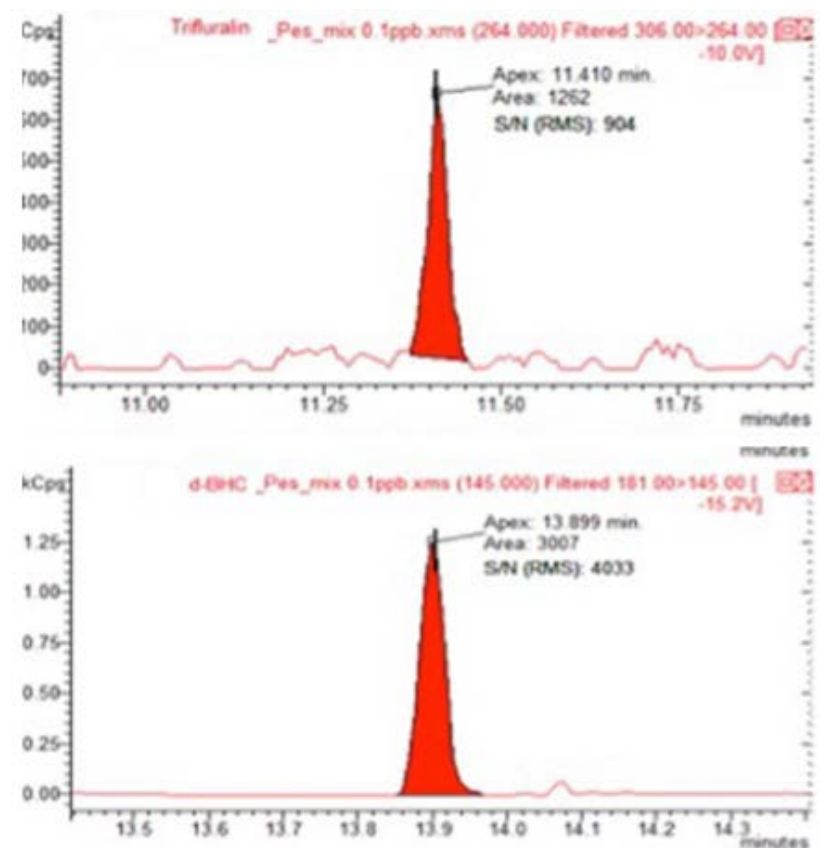

Figure 3. Peak of trifluralin and d-BHC at $0.001 \mathrm{mg} \mathrm{kg}^{-1}$ with RT (min), area and signal/noise ratio (both in counts per second)

The application of GC connected to tandem mass spectrometry (GC-MS/MS) in multi-class pesticide residue analysis has made the multi-residue analysis more 
rugged and convenient by offering the possibility of simultaneous determination of a large number of pesticides with varied physico-chemical properties without the need for chromatographic baseline separation. It has minimized the requirement for extensive sample cleanup, which was otherwise essential in earlier methods of analysis. The use of MRM mode removed the interfering peaks in the data acquisition and integration process. As a result, background noise was reduced and no peak was found missing due to interactions with matrix components. The use of this ethyl acetate extraction followed by a single-step clean-up and GC-MS/MS detection procedure could reliably identify pesticides at $0.001 \mathrm{mgkg}^{-1}$ (Figure 3).

Table 1. Different optimized GC-MS/MS parameters used for analysis of grapes and pomegranate and their validation results

\begin{tabular}{|c|c|c|c|c|c|c|c|c|c|}
\hline Pesticide & $\begin{array}{c}\text { RT } \\
\text { (min) }\end{array}$ & MRM/CE (V) & $\begin{array}{l}\text { Scan time } \\
(\mathrm{ms})\end{array}$ & $\mathbf{r}^{2}$ & $\begin{array}{c}\text { LOD (mg } \\
\left.\mathrm{kg}^{-1}\right)\end{array}$ & $\begin{array}{c}\text { LOQ (mg } \\
\left.\mathrm{kg}^{-1}\right)\end{array}$ & $\begin{array}{c}\% \\
\text { recovery }\end{array}$ & $\begin{array}{c}\text { Inter-day } \\
\text { precision(\%) }\end{array}$ & $\begin{array}{c} \pm \mathrm{UM} \\
(\%)\end{array}$ \\
\hline Hexaflumuron & 8.08 & $\begin{array}{l}202>110(-25) \\
202>174(-10)\end{array}$ & 30 & 0.992 & 0.0015 & 0.005 & $84-103$ & 12 & 18 \\
\hline Trifluralin & 11.416 & $\begin{array}{l}306>160 /(-25) \\
306>264 /(-10)\end{array}$ & 45 & 0.996 & 0.0015 & 0.005 & $89-102$ & 11.3 & 19 \\
\hline Phorate & 11.835 & $\begin{array}{c}121>93 /(-5) \\
260>75 /(-10) \\
\end{array}$ & 45 & 0.994 & 0.0015 & 0.005 & $87-105$ & 9 & 16 \\
\hline$\alpha-\mathrm{HCH}$ & 11.951 & $\begin{array}{l}181>145 /(-15) \\
219>183 /(-10)\end{array}$ & 30 & 0.993 & 0.0005 & 0.005 & $86-103$ & 9.6 & 17 \\
\hline Thiometon & 12.204 & $\begin{array}{l}125>47 /(-15) \\
125>79 /(-10)\end{array}$ & 75 & 0.996 & 0.0015 & 0.005 & $85-106$ & 8.9 & 14 \\
\hline Dimethoate & 12.347 & $\begin{array}{l}125>79 /(-10) \\
229>87 /(-10)\end{array}$ & 30 & 0.997 & 0.0015 & 0.005 & $87-107$ & 10.5 & 13 \\
\hline Carbofuran & 12.575 & $\begin{array}{l}164>103 /(-25) \\
164>149 /(-10)\end{array}$ & 30 & 0.995 & 0.0015 & 0.005 & $88-108$ & 11.2 & 19 \\
\hline Simazine & 12.626 & $\begin{array}{l}201>173 /(-5) \\
201>186 /(-5)\end{array}$ & 45 & 0.992 & 0.0015 & 0.005 & $94-103$ & 10.2 & 13 \\
\hline$\beta-\mathrm{HCH}$ & 12.712 & $\begin{array}{l}181>145 /(15.1) \\
219>183 /(10.1)\end{array}$ & 45 & 0.997 & 0.0005 & 0.005 & $94-106$ & 11.5 & 15 \\
\hline$\gamma-\mathrm{HCH}$ & 13.008 & $\begin{array}{l}181>145 /(-15) \\
219>183 /(-10)\end{array}$ & 60 & 0.999 & 0.0001 & 0.005 & $85-107$ & 11.7 & 16 \\
\hline Phosphamidon & 13.471 & $\begin{array}{l}264>127 /(-15) \\
264>193 /(-10)\end{array}$ & 45 & 0.999 & 0.0015 & 0.005 & $88-106$ & 10.7 & 15 \\
\hline Diazinon & 13.486 & $\begin{array}{l}179>137 /(-15) \\
304>179 /(-10) \\
\end{array}$ & 60 & 0.992 & 0.0015 & 0.005 & $88-104$ & 9.8 & 14 \\
\hline Chlorothalonil & 13.531 & $\begin{array}{l}266>133 /(-35) \\
266>231 /(-15) \\
\end{array}$ & 45 & 0.993 & 0.0015 & 0.005 & $87-105$ & 9.4 & 15 \\
\hline$\delta$-HCH & 13.902 & $\begin{array}{l}181>145 /(15.2) \\
219>183 /(10.2)\end{array}$ & 45 & 0.994 & 0.0005 & 0.005 & $87-108$ & 10.3 & 14 \\
\hline Formothion & 14.433 & $\begin{array}{l}170>93 /(-10) \\
125>93 /(-10) \\
\end{array}$ & 30 & 0.995 & 0.0015 & 0.005 & $86-106$ & 8.9 & 12 \\
\hline Metribuzin & 15.003 & $\begin{array}{c}198>82 /(-15) \\
198>110 /(-10)\end{array}$ & 75 & 0.997 & 0.0015 & 0.005 & $85-109$ & 8.8 & 12 \\
\hline $\begin{array}{c}\text { Methyl } \\
\text { parathion }\end{array}$ & 15.195 & $\begin{array}{c}263>109 /(-15) \\
263>246 /(-5)\end{array}$ & 30 & 0.995 & 0.0015 & 0.005 & $88-107$ & 9.6 & 13 \\
\hline Alachlor & 15.28 & $\begin{array}{l}160>132 /(-10) \\
188>160 /(-10)\end{array}$ & 45 & 0.999 & 0.0005 & 0.005 & $84-108$ & 7.6 & 11 \\
\hline Paraxon & 15.664 & $\begin{array}{l}149>91(-10) \\
149>119(-5)\end{array}$ & 90 & 0.994 & 0.0015 & 0.005 & $88-107$ & 5.7 & 10 \\
\hline $\begin{array}{c}\text { Pirimiphos } \\
\text { methyl }\end{array}$ & 16.07 & $\begin{array}{l}290>125 /(-20) \\
290>151 /(-20)\end{array}$ & 30 & 0.993 & 0.0015 & 0.005 & $85-107$ & 8.9 & 13 \\
\hline Fenarimol & 16.088 & $\begin{array}{l}277>109 /(-20) \\
277>260 /(-10)\end{array}$ & 45 & 0.992 & 0.0015 & 0.005 & $89-108$ & 6.8 & 11 \\
\hline Linuron & 16.295 & $\begin{array}{c}160>124 /(-10) \\
248>61 /(-10)\end{array}$ & 75 & 0.994 & 0.0015 & 0.005 & $91-105$ & 10.3 & 18 \\
\hline Malathion & 16.477 & $\begin{array}{l}173>99 /(-15) \\
173>127 /(-5)\end{array}$ & 30 & 0.992 & 0.0015 & 0.005 & $88-103$ & 12 & 17 \\
\hline Metolachlor & 16.59 & $\begin{array}{l}162>133 /(-15) \\
238>162 /(-15) \\
\end{array}$ & 45 & 0.995 & 0.0015 & 0.005 & $87-108$ & 9.7 & 13 \\
\hline Aldrine & 16.673 & $\begin{array}{l}263>193 /(-35) \\
263>228 /(-10)\end{array}$ & 30 & 0.994 & 0.0015 & 0.005 & $86-108$ & 9.7 & 14 \\
\hline Chlorpyrifos & 16.673 & $\begin{array}{l}314>258 /(-15) \\
314>286 /(-10)\end{array}$ & 45 & 0.995 & 0.0015 & 0.005 & 89-109 & 10.2 & 15 \\
\hline Fenthion & 16.8 & $\begin{array}{l}278>109 /(-20) \\
278>125 /(-20) \\
\end{array}$ & 30 & 0.996 & 0.0015 & 0.005 & $85-106$ & 7.7 & 14 \\
\hline Parathion & 16.927 & $\begin{array}{l}291>109 /(-15) \\
291>137 /(-10) \\
\end{array}$ & 30 & 0.993 & 0.0015 & 0.005 & $86-108$ & 7.8 & 15 \\
\hline Triadimefon & 17.053 & $\begin{array}{l}208>111 /(-20) \\
208>181 /(-10)\end{array}$ & 45 & 0.995 & 0.0015 & 0.005 & $87-102$ & 8.5 & 14 \\
\hline Pendimethalin & 17.839 & $\begin{array}{l}252>162 /(-10) \\
252>191 /(-10)\end{array}$ & 45 & 0.992 & 0.0015 & 0.005 & $84-103$ & 8.7 & 13 \\
\hline
\end{tabular}




\begin{tabular}{|c|c|c|c|c|c|c|c|c|c|}
\hline Pesticide & $\begin{array}{c}\mathrm{RT} \\
(\mathrm{min})\end{array}$ & MRM/CE (V) & $\begin{array}{c}\text { Scan time } \\
(\mathrm{ms})\end{array}$ & $\mathbf{r}^{2}$ & $\begin{array}{c}\text { LOD (mg } \\
\left.\mathrm{kg}^{-1}\right)\end{array}$ & $\begin{array}{c}\text { LOQ (mg } \\
\left.\mathrm{kg}^{-1}\right)\end{array}$ & $\begin{array}{c}\% \\
\text { recovery }\end{array}$ & $\begin{array}{c}\text { Inter-day } \\
\text { precision(\%) }\end{array}$ & $\begin{array}{c} \pm \text { UM } \\
(\%)\end{array}$ \\
\hline Penconazole & 18.033 & $\begin{array}{l}248>157 /(-25) \\
248>192 /(-15)\end{array}$ & 30 & 0.994 & 0.0015 & 0.005 & $85-105$ & 14.8 & 19 \\
\hline $\begin{array}{l}\text { Heptachlor } \\
\text { epoxide }\end{array}$ & 18.049 & $\begin{array}{l}353>263 /(-15) \\
353>282 /(-15)\end{array}$ & 45 & 0.993 & 0.001 & 0.005 & $88-102$ & 11.4 & 16 \\
\hline $\begin{array}{l}\text { Chlorienvinphos } \\
-\alpha\end{array}$ & 18.233 & $\begin{array}{l}267>159(-20) \\
323>267(-15)\end{array}$ & 30 & 0.994 & 0.0015 & 0.005 & $94-103$ & 10.7 & 15 \\
\hline Captan & 18.355 & $\begin{array}{l}149>79 /(-10.1) \\
149>105 /(-10)\end{array}$ & 30 & 0.996 & 0.0015 & 0.005 & $88-105$ & 10.4 & 14 \\
\hline Phenthoate & 18.39 & $\begin{array}{l}274>121 /(-10) \\
274>246 /(-10)\end{array}$ & 30 & 0.994 & 0.0015 & 0.005 & $89-104$ & 11.9 & 16 \\
\hline Quinalphos & 18.413 & $\begin{array}{l}146>118 /(-20) \\
157>129 /(-15)\end{array}$ & 30 & 0.994 & 0.0015 & 0.005 & $94-102$ & 11.3 & 17 \\
\hline Dieldrin & 18.888 & $\begin{array}{l}263>193 /(-25) \\
263>228 /(-20) \\
\end{array}$ & 60 & 0.995 & 0.001 & 0.005 & $86-101$ & 9.8 & 14 \\
\hline Chlordane - II & 18.888 & $\begin{array}{l}373>266 /(-20) \\
373>301 /(-10)\end{array}$ & 30 & 0.992 & 0.001 & 0.005 & $86-107$ & 11.5 & 17 \\
\hline O, P DDE & 19.048 & $\begin{array}{l}246>176 /(-30) \\
318>246 /(-25) \\
\end{array}$ & 75 & 0.996 & 0.001 & 0.005 & $88-107$ & 11.3 & 16 \\
\hline Butachlor & 19.325 & $\begin{array}{l}176>147 /(-15) \\
237>160 /(-10) \\
\end{array}$ & 75 & 0.992 & 0.001 & 0.005 & $89-106$ & 9.8 & 13 \\
\hline Endosulfan - $\alpha$ & 19.333 & $\begin{array}{l}241>170 /(-25) \\
241>206 /(-15) \\
\end{array}$ & 30 & 0.992 & 0.001 & 0.005 & $92-108$ & 9.6 & 15 \\
\hline Chlordane - I & 19.351 & $\begin{array}{l}373>266 /(-20) \\
373>301 /(-10)\end{array}$ & 60 & 0.994 & 0.001 & 0.005 & $84-106$ & 8.9 & 14 \\
\hline Hexaconazole & 19.858 & $\begin{array}{l}214>152 /(-20) \\
214>159 /(-20)\end{array}$ & 30 & 0.996 & 0.0015 & 0.005 & $90-105$ & 8.9 & 12 \\
\hline Tricyclazole & 19.88 & $\begin{array}{l}162>134 /(-20) \\
189>161 /(-15) \\
\end{array}$ & 90 & 0.992 & 0.0015 & 0.005 & $91-108$ & 7.8 & 11 \\
\hline Pretilachlor & 20.066 & $\begin{array}{l}162>147 /(-15) \\
176>134 /(-15) \\
\end{array}$ & 75 & 0.994 & 0.0015 & 0.005 & $89-107$ & 6.7 & 11 \\
\hline Profenofos & 20.105 & $\begin{array}{c}139>97 /(-10) \\
339>269 /(-15)\end{array}$ & 30 & 0.993 & 0.0015 & 0.005 & $87-105$ & 7.5 & 12 \\
\hline P, P DDE & 20.229 & $\begin{array}{l}246>176 /(-20) \\
246>211 /(-20)\end{array}$ & 60 & 0.993 & 0.001 & 0.005 & $89-109$ & 9.4 & 14 \\
\hline Fenitrothion & 20.238 & $\begin{array}{l}383>255 /(-20) \\
383>335 /(-10)\end{array}$ & 60 & 0.997 & 0.0015 & 0.005 & $85-107$ & 5.4 & 11 \\
\hline Oxadiazon & 20.357 & $\begin{array}{l}258>112 /(-25) \\
258>175 /(-10) \\
\end{array}$ & 30 & 0.995 & 0.0015 & 0.005 & $84-109$ & 4.4 & 10 \\
\hline O, P DDD & 20.433 & $\begin{array}{l}235>165 /(-25) \\
235>199 /(-15)\end{array}$ & 60 & 0.995 & 0.001 & 0.005 & $84-106$ & 8.9 & 13 \\
\hline Buprofezin & 20.541 & $\begin{array}{l}172>115 /(-10) \\
249>193 /(-10)\end{array}$ & 60 & 0.993 & 0.0015 & 0.005 & $88-108$ & 7.9 & 13 \\
\hline Oxyfluorfen & 20.57 & $\begin{array}{l}252>146 /(-25) \\
302>274 /(-15)\end{array}$ & 30 & 0.996 & 0.0015 & 0.005 & $86-103$ & 9.8 & 15 \\
\hline Endosulfan - $\beta$ & 21.399 & $\begin{array}{l}195>159 /(-10) \\
241>206 /(-15) \\
\end{array}$ & 75 & 0.998 & 0.0015 & 0.005 & $88-98$ & 10.5 & 15 \\
\hline P, P DDD & 21.705 & $\begin{array}{l}235>165 /(-15) \\
235>199 /(-15)\end{array}$ & 30 & 0.994 & 0.001 & 0.005 & $94-101$ & 10.8 & 16 \\
\hline Ethion & 21.776 & $\begin{array}{l}231>129 /(-25) \\
231>175 /(-10)\end{array}$ & 75 & 0.993 & 0.0015 & 0.005 & $85-101$ & 10.2 & 16 \\
\hline DDT - OP & 21.776 & $\begin{array}{l}235>165 /(15.1) \\
235>199 /(15.1)\end{array}$ & 45 & 0.992 & 0.001 & 0.005 & $93-107$ & 11.2 & 17 \\
\hline $\begin{array}{c}\text { Endosulfan } \\
\text { sulfate }\end{array}$ & 22.783 & $\begin{array}{l}272>237 /(-15) \\
387>253 /(-10)\end{array}$ & 30 & 0.996 & 0.0015 & 0.005 & $84-103$ & 9.4 & 13 \\
\hline Propiconazole & 23.041 & $\begin{array}{c}173>145 /(-15) \\
259>69 /(-10)\end{array}$ & 75 & 0.994 & 0.0015 & 0.005 & $87-102$ & 13.8 & 18 \\
\hline DDT - PP & 23.049 & $\begin{array}{l}235>165 /(-15) \\
235>200 /(-10) \\
\end{array}$ & 45 & 0.997 & 0.001 & 0.005 & $88-106$ & 7.8 & 15 \\
\hline Heptachlor & 23.225 & $\begin{array}{l}171>71 /(-15) \\
171>85 /(-15)\end{array}$ & 45 & 0.992 & 0.001 & 0.005 & $89-106$ & 9.8 & 14 \\
\hline Tebuconazole & 23.532 & $\begin{array}{l}250>125 /(-10) \\
250>163 /(-10)\end{array}$ & 75 & 0.993 & 0.0015 & 0.005 & $85-108$ & 13.6 & 19 \\
\hline Diclofop-methyl & 23.681 & $\begin{array}{l}253>162 /(-15) \\
340>253 /(-10)\end{array}$ & 90 & 0.995 & 0.0015 & 0.005 & $84-105$ & 9.8 & 14 \\
\hline Iprodione & 24.565 & $\begin{array}{l}314>245 /(-15) \\
314>271 /(-10) \\
\end{array}$ & 45 & 0.992 & 0.0015 & 0.005 & $88-102$ & 10.9 & 13 \\
\hline Bifenthrin & 24.968 & $\begin{array}{l}181>141 /(-20) \\
181>166 /(-15)\end{array}$ & 60 & 0.993 & 0.0015 & 0.005 & $92-101$ & 11.2 & 15 \\
\hline Fenpropathrin & 25.264 & $\begin{array}{l}181>152 /(-20) \\
265>210 /(-15)\end{array}$ & 75 & 0.996 & 0.0015 & 0.005 & $86-103$ & 10.6 & 14 \\
\hline
\end{tabular}




\begin{tabular}{|c|c|c|c|c|c|c|c|c|c|}
\hline Pesticide & $\begin{array}{c}\text { RT } \\
\text { (min) }\end{array}$ & MRM/CE (V) & $\begin{array}{c}\text { Scan time } \\
(\mathrm{ms})\end{array}$ & $\mathbf{r}^{2}$ & $\begin{array}{c}\text { LOD (mg } \\
\left.\mathrm{kg}^{-1}\right)\end{array}$ & $\begin{array}{c}\text { LOQ (mg } \\
\left.\mathbf{k g}^{-1}\right)\end{array}$ & $\begin{array}{c}\% \\
\text { recovery }\end{array}$ & $\begin{array}{c}\text { Inter-day } \\
\text { precision(\%) }\end{array}$ & $\begin{array}{c} \pm \mathrm{UM} \\
(\%)\end{array}$ \\
\hline Phosalone & 25.987 & $\begin{array}{l}182>111 /(-15) \\
182>138 /(-10)\end{array}$ & 30 & 0.995 & 0.0015 & 0.005 & $86-104$ & 9.6 & 14 \\
\hline Cyhalothrin $\lambda$ & 26.893 & $\begin{array}{l}181>152 /(-20) \\
197>141 /(-15)\end{array}$ & 30 & 0.997 & 0.0015 & 0.005 & 89-98 & 8.5 & 14 \\
\hline Edifenphos & 26.985 & $\begin{array}{l}139>111 /(-15) \\
251>139 /(-15)\end{array}$ & 60 & 0.999 & 0.0015 & 0.005 & 88-106 & 8.7 & 13 \\
\hline Bitertanol & 28.137 & $\begin{array}{l}170>115 /(-30) \\
170>141 /(-20)\end{array}$ & 30 & 0.994 & 0.0015 & 0.005 & $92-107$ & 6.7 & 12 \\
\hline Permethrin I & 28.349 & $\begin{array}{c}163>127 /(-5) \\
183>153 /(-15) \\
\end{array}$ & 30 & 0.992 & 0.0015 & 0.005 & $94-108$ & 9.7 & 15 \\
\hline Permethrin II & 28.60 & $\begin{array}{l}163>127 /(-5.1) \\
183>153 /(15.1) \\
\end{array}$ & 60 & 0.993 & 0.0015 & 0.005 & $85-109$ & 10.4 & 16 \\
\hline b-Cyfluthrin & 29.642 & $\begin{array}{l}163>127 /(-5.1) \\
206>151 /(20.1) \\
\end{array}$ & 75 & 0.997 & 0.0015 & 0.005 & $92-103$ & 5.6 & 11 \\
\hline Cypermethrin I & 30.035 & $\begin{array}{c}163>127 /(-5) \\
181>152 /(-20) \\
\end{array}$ & 50 & 0.995 & 0.0015 & 0.005 & 89-101 & 9 & 14 \\
\hline Cypermethrin II & 30.25 & $\begin{array}{c}163>127 /(-5.1) \\
181>152 /(-20.1) \\
\end{array}$ & 50 & 0.995 & 0.0015 & 0.005 & $88-103$ & 10.8 & 13 \\
\hline $\begin{array}{c}\text { Cypermethrin } \\
\text { III } \\
\end{array}$ & 30.346 & $\begin{array}{c}163>127 /(-5.2) \\
181>152 /(-20.2) \\
\end{array}$ & 30 & 0.993 & 0.0015 & 0.005 & $89-108$ & 11.2 & 15 \\
\hline $\begin{array}{c}\text { Cypermethrin } \\
\text { IV } \\
\end{array}$ & 30.439 & $\begin{array}{c}163>127 /(-5.3) \\
181>152 /(-20.3) \\
\end{array}$ & 30 & 0.996 & 0.0015 & 0.005 & $84-101$ & 11.7 & 16 \\
\hline Fenvalerate I & 31.718 & $\begin{array}{c}125>89 /(-20) \\
167>125 /(-10) \\
\end{array}$ & 30 & 0.994 & 0.0015 & 0.005 & $85-104$ & 9.7 & 14 \\
\hline Fluvalinate & 32.029 & $\begin{array}{c}250>55 /(-10) \\
250>200 /(-15) \\
\end{array}$ & 45 & 0.993 & 0.0015 & 0.005 & $86-92$ & 8.9 & 13 \\
\hline Esfenvalerate & 32.122 & $\begin{array}{c}125>89 /(-20) \\
167>125 /(-10) \\
\end{array}$ & 45 & 0.995 & 0.0015 & 0.005 & $91-109$ & 11 & 15 \\
\hline Fenvalerate II & 32.127 & $\begin{array}{c}125>89 /(-20.1) \\
167>125 /(-10.1) \\
\end{array}$ & 45 & 0.992 & 0.0015 & 0.005 & $89-106$ & 10.7 & 15 \\
\hline Difenoconazole & 32.626 & $\begin{array}{l}323>265 /(-15) \\
323>202 /(-25)\end{array}$ & 45 & 0.995 & 0.0015 & 0.005 & $88-101$ & 14.6 & 19 \\
\hline Deltamethrin & 33.158 & $\begin{array}{c}253>93 /(-20) \\
253>172 /(-10) \\
\end{array}$ & 45 & 0.992 & 0.0015 & 0.005 & $90-102$ & 12 & 16 \\
\hline
\end{tabular}

Compound name, Retention time (min), MRM transition with precursor and product ions and their applied Collision Energy (volt), Dwell time (ms),

Limit of detection and quantification ((LOD and LOQ in $\mathrm{mgkg}^{-1}$ ), coefficient of regression (r2), Recoveries ( \%) (Average range in 2 commodities),

Repeatability Inter-day precision expressed as \% RSD and overall uncertainties expressed as \% $(\mathrm{k}=2)$ calculated at LOQ level.

GC-MS/MS was operated in the MRM mode due to its the EU legislation. Briefly, the EU Commission's decision high specificity and capability of simultaneously monitoring numerous product ions of a large number of compounds in complex sample matrices. Therefore optimization began with the GC-MS/MS data acquisition parameters; optimization of precursor and product ions and collision energies (CE). The precursor ions, product ions and collision energies optimized for all 82 pesticides are tabulated in Table 1. Suitable MRM transitions for each compound were selected carefully to ensure specificity and sensitivity of the determination. Initially, the complete precursor and product ion scan for each pesticide was investigated and then the collision energies were optimized to obtain the best response for two selected product ions. Theoretically, the best option for the choice of precursor ion for MS/MS fragmentation is the base ion in the mass spectrum as it shows the highest intensity. But in practice, this ion does not necessarily represent the best choice, as usually even less intensive ions in the high mass range are usually more adequate in terms of specificity and higher signal-to-noise ratio. After considering all these factors, appropriate MS/MS MRM transitions were optimized, without a significant trade-off between sensitivity and selectivity. However, quantification was always done using one transition while the second transition served for unequivocal identification (confirmation) of detected pesticide residues. Results were regarded as positively identified when signal-to-noise ratio (S/N) was $>3$ for both transitions and RSD of the respective ion ratios fell within the tolerances specified in in 2002 implements the concept of identification points (IPs) where 1 identification point is earned from a precursor ion, and 1.5 identification points are earned from a resulting product ion. For the unequivocal confirmation of a compounds' identity, at least 3 and 4 identification points are required for legal and banned substances, respectively. In this work, two product ions resulting from fragmentation of one precursor ion or two product ions each resulting from two different precursor ions were monitored. Hence, 4 or 5 identification points were acquired for the majority of compounds.

Secondly, for all studied analytes, the ion ratio for each MRM (ratios between $20 \%$ and $50 \%$ of the most intense transition) matches with the certified reference material within specified tolerances of $\pm 25 \%$.The use of CBS significantly simplifies development of MRM and processing of data by automatically linking data. This eliminates the need for separate method set-up, quantification and confirmation. Based on the elution profile of pesticides, a time-scheduled acquisition method was constructed, called "time segments" by CBS software.

The present scope of the method covers 82 compounds and a total of 184 MRM transitions (including crossedover transitions) monitored with 109 RT-segments (2-3 transitions each). Figure 4 shows RT-windows (segments) of a spiked grape samples where some overlap of MRM transitions from one time-segment to another was divided automatically. 


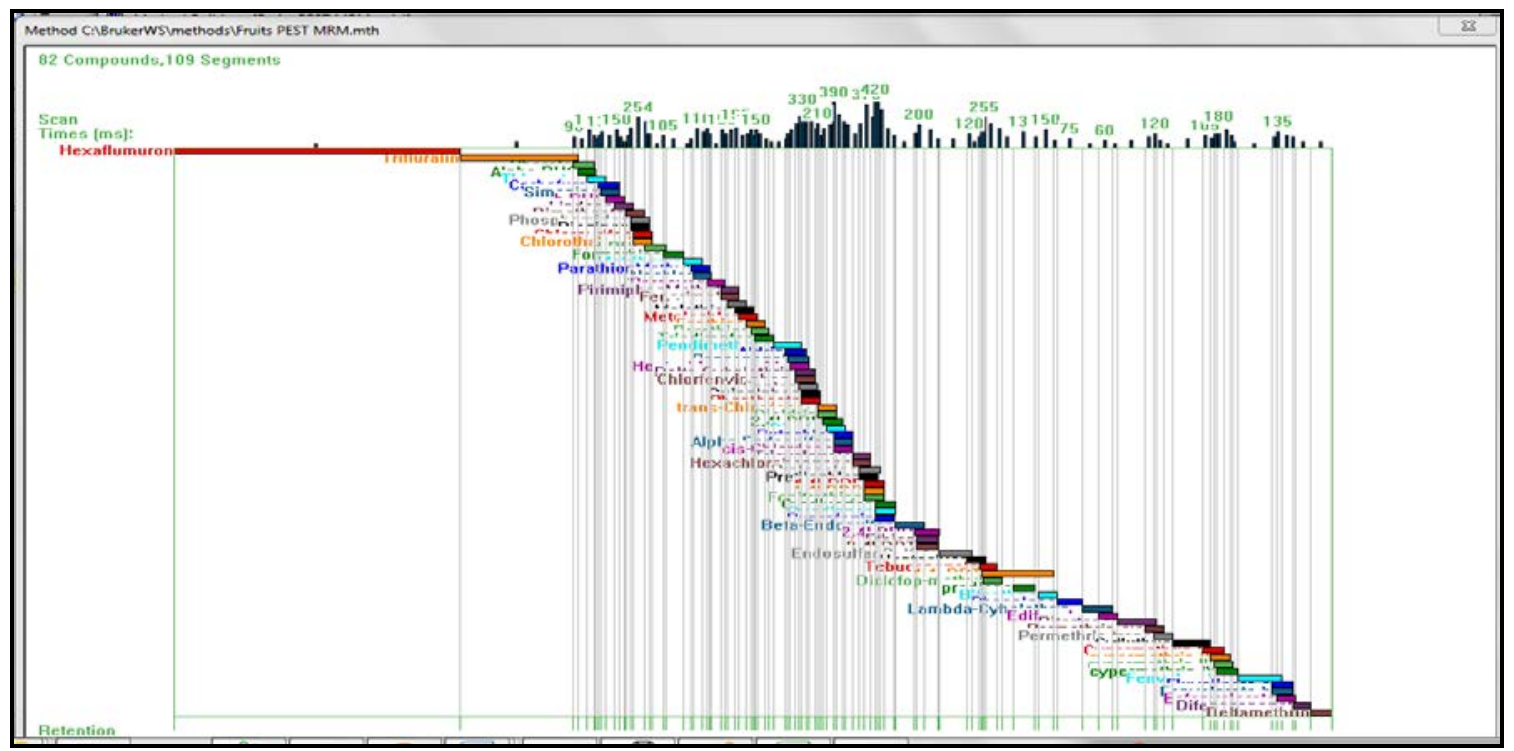

Figure 4. The RT-windows (segments) of spiked grapes (82 pesticides in 109 segments)

Thus, the transitions of critical compounds eluting close to the beginning or the end of adjacent time-segments were acquired in two segments to facilitate integration and prevent target compounds from being missed. The scan time for each compound (each with 2 MRMs) was optimized between 30 to $90 \mathrm{~ms}$ to satisfy sensitivity by considering sufficient data points $(>15)$ across each peak (Figure 5).

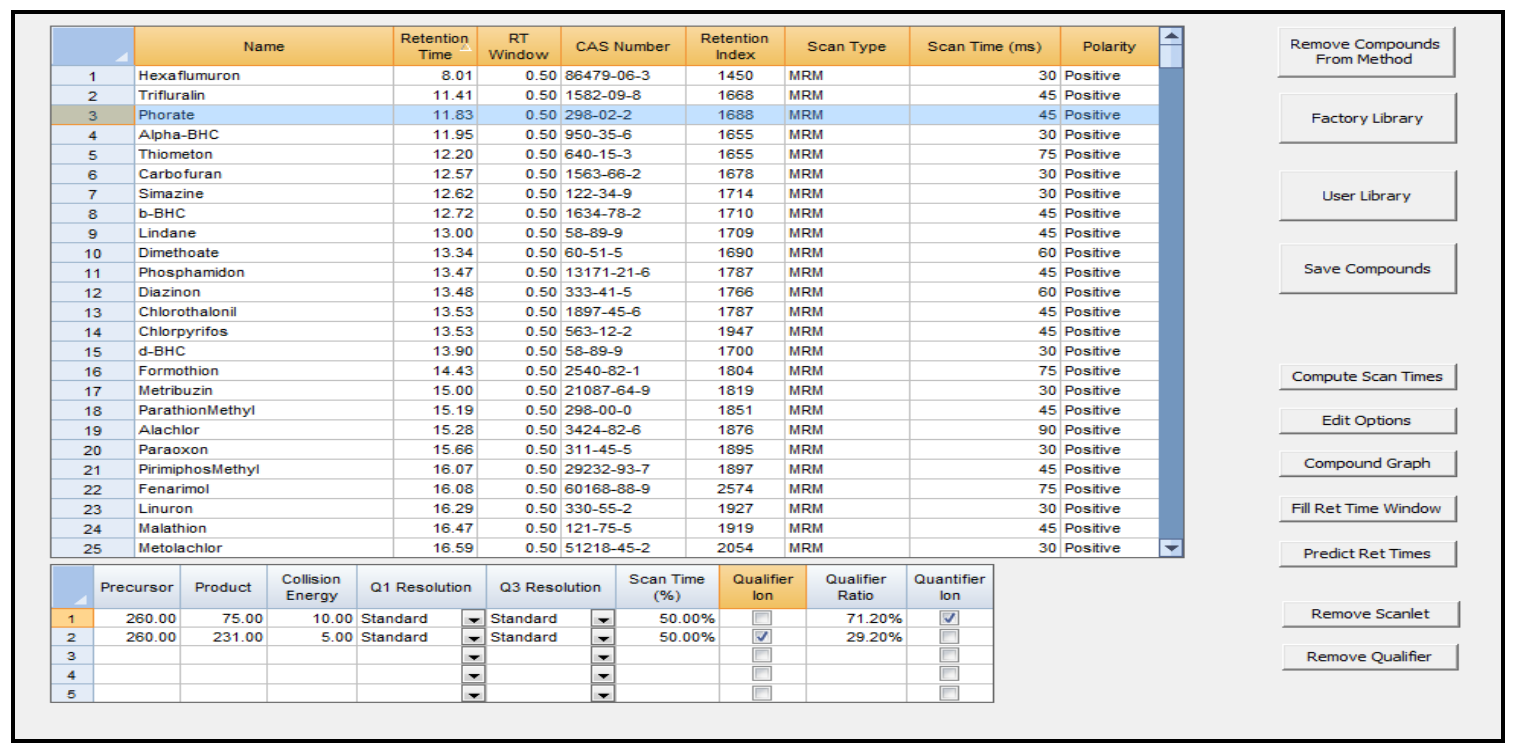

Figure 5. The CBS window for GC-MS/MS-MRM optimization (retention time, scan time, qualifier and quantifier ions and collision energy)

\subsection{Method Performance}

The combination of pesticides and foods which were tested, were determined by the potential of the analytical methods and the health risks to consumers due to pesticide contamination. Keeping this in mind, the method should be robust, give accurate results, meet detection limit needs, and cover the desired scope of matrices and analytes. Eventually, the developed method was validated in terms of linearity, recoveries and precision. All quantitative results were calculated using matrix-matched standards prepared by spiking extracts of the original grapes and pomegranate samples with different levels of pesticides as recommended by the European guidelines. Linearity was studied in matrix-matched standard solutions of grapes and pomegranate separately. All 82 pesticides belonging to various classes were separated and detected between
$5.95 \mathrm{~min}$ and $33.2 \mathrm{~min}$. Fig.6 shows the spiked grape sample with 28 pesticides at $0.005 \mathrm{mgkg}^{-1}$ level.

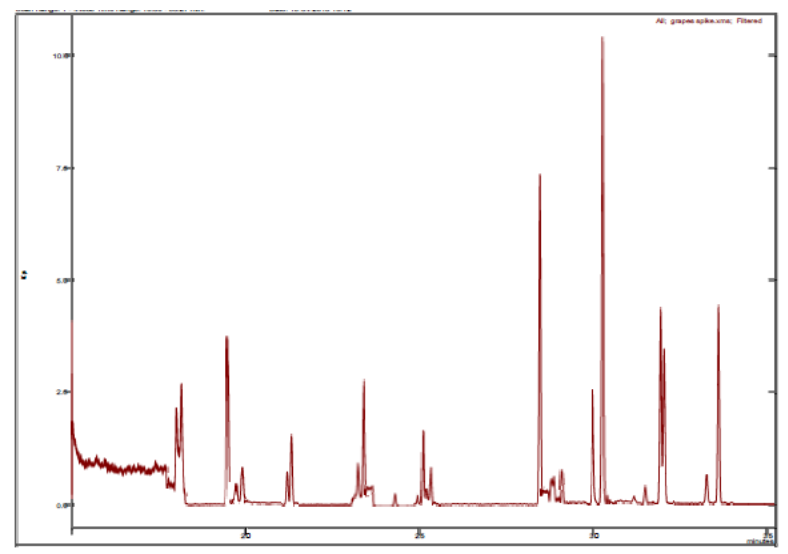

Figure 6. Spiked grapes sample with 28 pesticides at $0.005 \mathrm{mgkg}^{-1}$ level 
Good linearity of the calibration curves was obtained for all compounds analysed by GC-MS/MS over the entire range of $0.005-0.1 \mathrm{mgkg}^{-1}$ evaluated by duplicate analyses at five different concentration levels. The target compounds showed coefficients of regression $\left(r^{2}\right)$ more than 0.99 for all compounds (Figure 7).

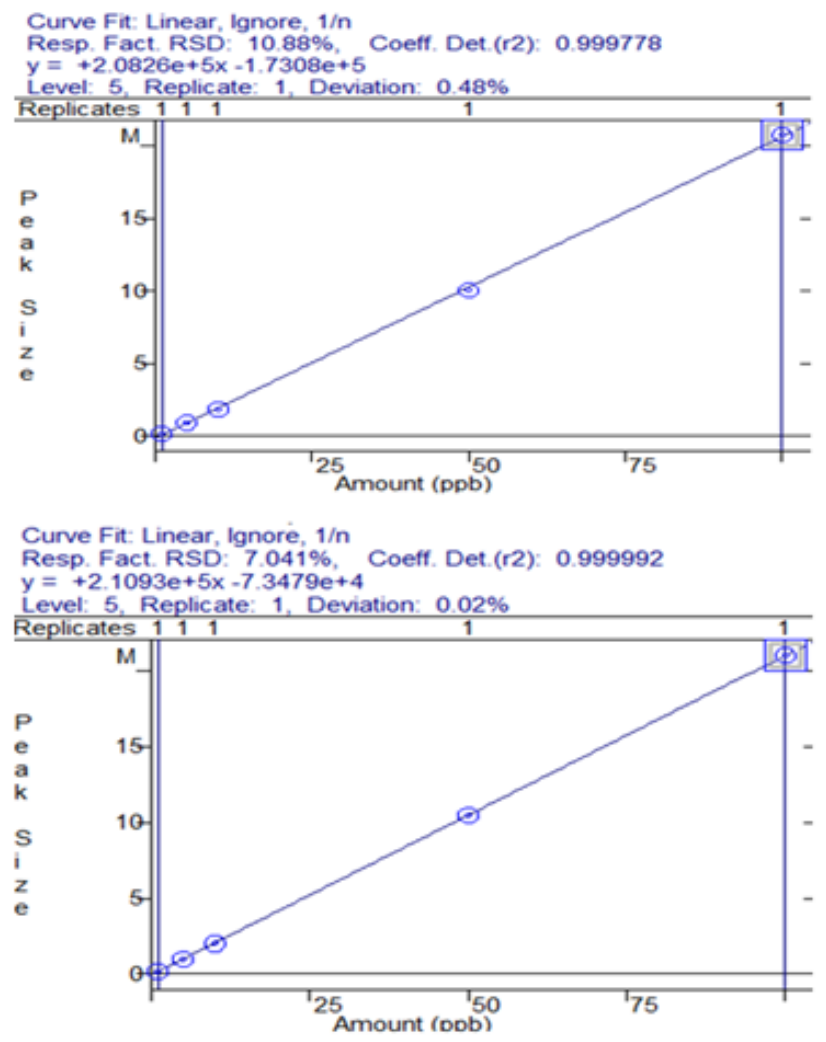

Figure 7. Calibration curve (0.005 to $0.1 \mathrm{mg} \mathrm{kg}^{-1}$ ) of edifenphos (grapes) and alachlor (pomegranates) by GC-MS/MS

The analytical results obtained for the studied compounds are shown in Table 1.

Recovery experiments were carried out at three different spiking levels of $0.01,0.05$, and $0.1 \mathrm{mg} \mathrm{kg}^{-1}$. The lowest spiking concentration of $0.01 \mathrm{mg} \mathrm{kg}^{-1}$ was selected to test the method performance at the restrictive residue level set by EU legislation for fruits. UM was calculated as per the ISO guide [15]. In the present work, the overall uncertainties were calculated at $0.01 \mathrm{mg} \mathrm{kg}^{-1}$ level (Table 1). These were calculated from the $n=20$ results (each matrix) from the experiment performed under repeatable and reproducible conditions. The obtained results demonstrated that the method achieved satisfactory quantitative recoveries in the range of $84-109 \%$ with RSD $<12 \%$ for repeatability and intermediate precision and UM of \pm 12 - $19 \%$. The present study also included the analytes; penconazole, tricyclazole, difenoconazole, hexaconazole, tebuconazole, carbofuran and oxyfluorfen, which are nowadays analyzed by LC-MS/MS $[17,18]$ to evaluate the usefulness of GC-MS/MS for their determination.

Analytical quality control was applied in order to assure the quality of the obtained results and identify limitations of the method. All sets of samples were accompanied by analysis of matrix blanks (if available for the particular matrix), spiked samples and matrix-matched calibration standards bracketing the samples. Method performance data was usually acquired by analysing spiking recovery at
$0.01 \mathrm{mgkg}^{-1}$. For this purpose, randomly selected real samples (both grapes and pomegranate) were used; as a result, somewhat more recoveries within the range of $70 \%$ and $120 \%$ were obtained, because of some pesticide residues already present in the sample. In such cases the subsequent calibrations were all carried out with pesticides spiked in the fruit matrices. As a result, the RSD for average response of the pesticides over the entire calibration range were obtained within the limit (RSD $\leq$ $12 \%)$, thus proving the excellent precision of the method for analysing these pesticides in a complex sample matrix (Figure 8).

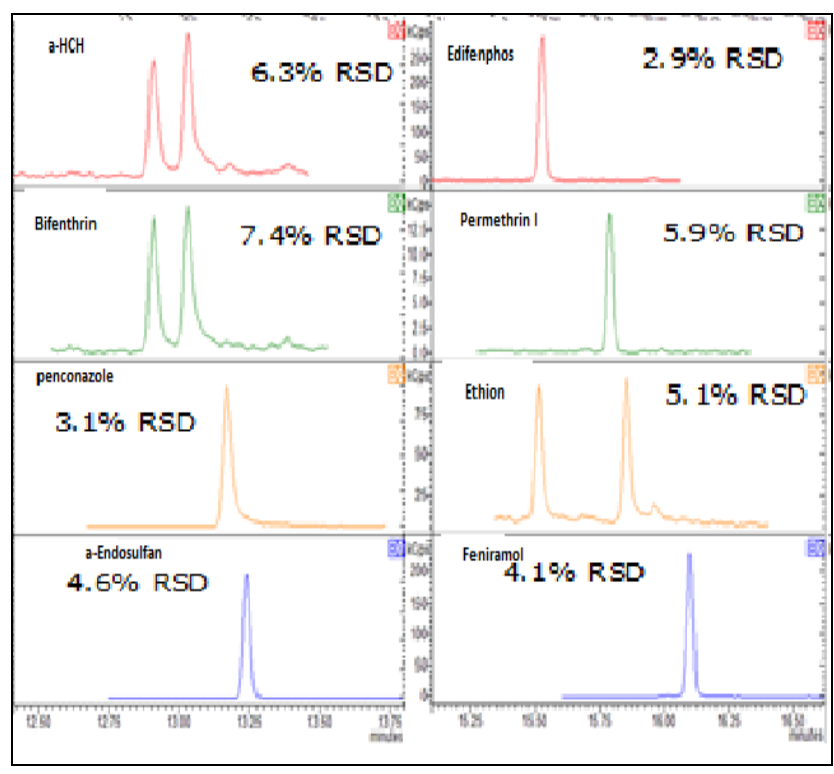

Figure 8. Precision of different group of pesticides in grapes $(n=5) ; \alpha$ $\mathrm{HCH}$, edifenphos, bifenthrin, permethrine I, penconazole, ethion, $\alpha$ endosulfan and feniramol

\section{Conclusion}

The proposed method using single step SLE with ethyl acetate followed by PSA and GCB clean-up has greatly simplified the method for multiresidue pesticide analysis and also improved the productivity for all non-polar GC grade pesticides. Contrary to single-stage MS technique (such as full scan and SIM), the MS/MS produces more unique spectra resulting from two consecutive steps of mass fragmentation and it helps to solve analytical problems where the single-stage MS techniques fail. This work has demonstrated that GC-MS/MS is a key tool for comprehensive multi-residue screening of pesticides belonging to various chemical classes in complex matrices such as grapes and pomegranate. The method has shown good sensitivity, linear calibration range, robustness in the matrix, and ability to meet the requirement of EU Commission decision and quantitate the pesticides at the level of $0.01 \mathrm{mgkg}^{-1}$.

\section{References}

[1] APEDA, Regulation of export of fresh grapes from India through monitoring of pesticide residues, Amendments in grape RMP 2007, Amendment-5 (Revised Annexure - 7\&11), 2006a. Available: http://www.apeda.com. [Accessed Oct. 30, 2011]. 
[2] APEDA,Regulation of export of fresh grapes from India through monitoring of pesticide residues, Annexure 1: Circular No.91-4/95 PQD dated 29th February 2000, 2006b.

Available:http://www.apeda.com/apeda/GRAPES 05/ RMPGrapes 200717 oct06 3.pdf. [Accessed Oct. 30, 2011].

[3] Schachterle, S. andFeigel, C, "Pesticide residue analysis in fresh produce by gas chromatography-tandem mass spectrometry," Journal of Chromatography A, 754. 411-422.1996.

[4] Torres, C.M., Pico, Y. and Manes, J, "Determination of pesticide residues in fruit and vegetables - a review," Journal of Chromatography A, 754. 301-331. 1996.

[5] Herdman, R.C., Pahrham, W.E., and Shen, S,Pesticide Residues in Food: Technologies for Detection, Government Printing Office, Washington, 1988.

[6] Cajka, T.and Hajslová, J, "Gas chromatography-time-of-flight mass spectrometry in food analysis," $L C-G C$ Europe, 20(1). 25-26. 2007.

[7] Lambropoulou, D.A. and Albanis, T.A, "Methods of sample preparation for determination of pesticide residues in food matrices by chromatography-mass spectrometry-based techniques: a review," Analytical and Bioanalytical Chemistry, 389 (6). 166383.2007.

[8] van der Lee, M.K., van derWeg, G., Traag, W.A. and Mol, H.G.J, "Qualitative screening and quantitative determination of pesticides and contaminants in animal feed using comprehensive twodimensional gas chromatography with time-of-flight mass spectrometry," Journal of Chromatography A, 1186. 325.2008.

[9] M. Anastassiades, Recovery Studies using the QuEChERS Method, 2008. Available: http://www.quechers.com/. [Accessed Apr. 3, 2011].

[10] AOAC Official Method 2007.01. Pesticide Residues in Foods by Acetonitrile Extraction and Partitioning with Magnesium Sulfate.2007.

[11] Satpathy, G., Tyagi, Y.K. and Gupta, R.K, "A novel optimized and validated method for analysis of multi-residues of pesticides in fruits and vegetables by microwave-assisted extraction (MAE)dispersive solid-phase extraction (d-SPE)-retention time locked (RTL)-gas chromatography-mass spectrometry with Deconvolution reporting software (DRS)", Food Chemistry, 127. 1300-1308. 2011.

[12] Walorczyk, S, "Development of a multi-residue screening method for the determination of pesticides in cereals and dry animal feed using gas chromatography-triple quadrupole tandem mass spectrometry,” Journal of Chromatography A, 1165(1-2). 200-12. 2007.

[13] Mol, H.G.J., van Dam, R.C.J. and Steijger, O.M, “Determination of polar organophosphorus pesticides in vegetables and fruits using liquid chromatography with tandem mass spectrometry: selection of extraction solvent," Journal of Chromatography A, 1015. 119-127. 2003.

[14] Banerjee, K., Upadhyay, A.K., Adsule, P.G., Patil, S.H., Oulkar, D.P. and Jadhav, D.R, "Rate of degradation of lambda-cyhalothrin and methomyl in grapes (Vitisvinifera L.)," Food Additives and Contaminates, 23. 994-999.2006.

[15] GUM, ISO guide to the expression of uncertainty in measurement. BIPM, IEC, IFCC, ISO, IUPAC, IUPAP, OIML, Geneva. 1993.

[16] EURACHEM, Quantifying uncertainty in analytical measurements, EURACHEMSecretariat, P.O. Box 46, Teddington, Middlesex, TW110LY, UK, 1995.

[17] Hiemstra, M. and de Kok, A, "Comprehensive multi-residue method for the target analysis of pesticides in crops using liquid chromatography-tandem mass spectrometry," Journal of Chromatography A, 1154 (1-2). 3-25. 2007.

[18] Pizzutti, I.R., de Kok, A., Zanella, R., Adaime, M.B., Hiemstra, M., Wickert, C. and Prestes, O.D, "Method validation for the analysis of 169 pesticides in soya grain, without clean up, by liquid chromatography-tandem mass spectrometry using positive and negative electrospray ionization," Journal of Chromatography A, 1142(2). 123-36. 2007. 